\title{
Nuclotron Beam Momentum Estimation Using Multiwire Proportional Chambers and Drift Chambers in the BM@N Experiment
}

\author{
Mikhail Kapishin ${ }^{1}$, Vasilisa Lenivenko ${ }^{1}$, Vladimir Palichik ${ }^{1}$, and Nikolay Voytishin ${ }^{1, \star}$ \\ ${ }^{1}$ Joint Institute for Nuclear Research, Joliot Curie 6, 141980 Dubna, Moscow region, Russia
}

\begin{abstract}
.
The BM@N experiment is considered as the first phase of NICA Mega science project. The energy of the beam will vary from 1 to $6 \mathrm{GeV} / \mathrm{u}$. The beams delivered by Nuclotron will be of different types from protons to Au. The ability to reconstruct the beam momentum with high precision is one way for showing that the tracking detectors are tuned in the right way and the reconstruction procedure performs well.

A quick overview of the experimental setup is given in the work along with the description of some of the main tracking detectors. The beam momentum reconstruction procedure is described and results are presented for different values of the beam energy.
\end{abstract}

\section{Introduction}

The Baryonic Matter at Nuclotron (BM@N) is the first step of the realization of the Nuclotron-based Ion Collider fAcility (NICA) mega-science project [1]. The purpose of the BM@N experiment is to study the interactions of relativistic heavy ion beams delivered by the Nuclotron with fixed targets [2]. The Nuclotron facility is able to provide various types of beams with kinetic energies from 1 to $6 \mathrm{GeV}$ per nucleon. The experimental setup is a complex structure. Its main parts are the analyzing magnet, a set of tracking detectors and a set of identification detectors. All of them are meant to become a precise tool for the study of strange hyperon and hyper-nuclei production yields and ratios.

The first cycles of physical data gathering passed. The accuracy assessment of the main detector systems performance is one of the main tasks at the moment. This paper presents the results of the beam momentum reconstruction procedure using two tracking detector systems: Multi-Wire Proportional Chambers (MWPC) and Drift Chambers (DCH). The accuracy of this reconstruction provides a measure to the performance capabilities of these two detector systems.

\section{MWPC and DCH tracking systems}

The MWPC are situated in front of the analyzing magnet and are mostly used for the alignment of the entire experimental setup and for the incoming beam trajectory positioning. They represent standard proportional chambers [3]. They consist of three double coordinate planes with wires positioned at

\footnotetext{
^e-mail: voitishinn@gmail.com
} 
angles of $0^{\circ}$ and $\pm 60^{\circ}$. The wire pitch is $\mathrm{d}=2.5 \mathrm{~mm}$ and the estimated spatial resolution is $d / \sqrt{12}=$ $720 \mu \mathrm{m}$. The reconstruction algorithm for these chambers [4] was developed and implemented into the official experiment software package. The analysis of recent experimental data showed that the chambers reconstructed tracks with high efficiency. The beam alignment procedure was performed, providing the remaining part of the detector systems the beam position used for global alignment.

The DCH detectors were granted from the finalized NA48 experiment [5]. Two DCH are used in the experimental setup. They form the outer tracker. Each drift chamber consists of four double coordinate planes with wires positioned at angles of $0^{\circ}, 90^{\circ}$ and $\pm 45^{\circ}$. The wire pitch is $10 \mathrm{~mm}$. The spatial resolution achieved by using the experimental data varies from 180 to $300 \mu \mathrm{m}$ for different planes. The reconstruction efficiency for all planes is above $90 \%$. Good spatial resolution and high reconstruction efficiency shows that the work aimed at restoring the detectors to their initial state was done with a high degree of professionalism.

\section{Beam momentum estimation procedure}

The beam trajectory passing through the magnetic field is shown schematically in figure 1. Its momentum can be estimated out of the experimental data [6] by using the formula:

$$
\frac{P_{\text {beam }}}{Z_{\text {beam }}}=\frac{0.3 \cdot \int B d l}{\sin \left(\alpha_{\text {out }}-\alpha_{\text {in }}\right)},
$$

where $\int B d l$ is the magnetic field integral, $\alpha_{\text {in }}$ is the particle trajectory angle before the magnet given by MWPC, $\alpha_{\text {out }}$ is the particle trajectory angle after the magnet given by $\mathrm{DCH}$ and 0.3 is the coefficient for transferring Tesla $\cdot$ meter to $\mathrm{GeV} / \mathrm{c}$.

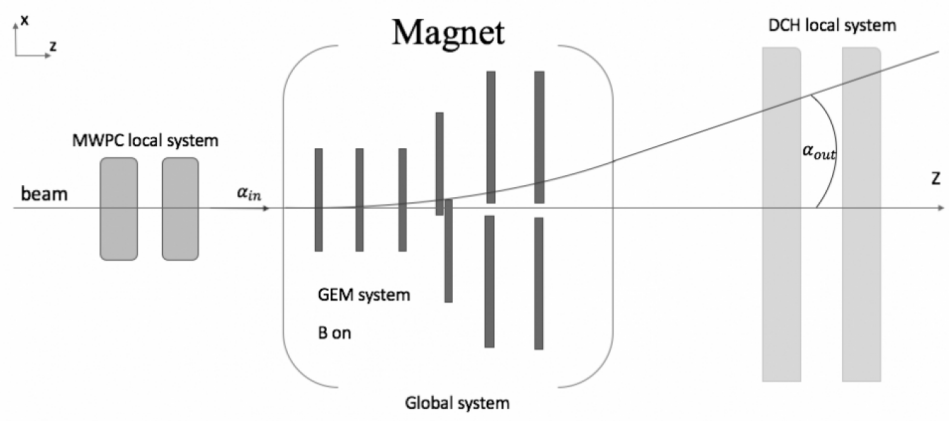

Figure 1. BM@N tracking system

The nominal beam momentum value can be established from the formula:

$$
\frac{P_{\text {beam }}}{Z_{\text {beam }}}=\frac{A}{Z} \sqrt{\left[E+M_{p}\right]^{2}-M_{p}^{2}},
$$

where $A$ is the mass number of the ion, $Z$ is the number of protons, $E$ is the beam kinetic energy per nucleon and $M_{p}$ is the proton mass. 


\section{Results and analysis}

The most recent experimental data were gathered with carbon beams with different energies. We considered two different values of energies of 3.5 and $4.5 \mathrm{GeV} /$ nucleon in this paper. The beam momentum was estimated for diverse values of the magnetic field integrals in order to get a complete illustration of the method performance.

The results for the $3.5 \mathrm{GeV} /$ nucleon carbon beam are presented on figure 2 .

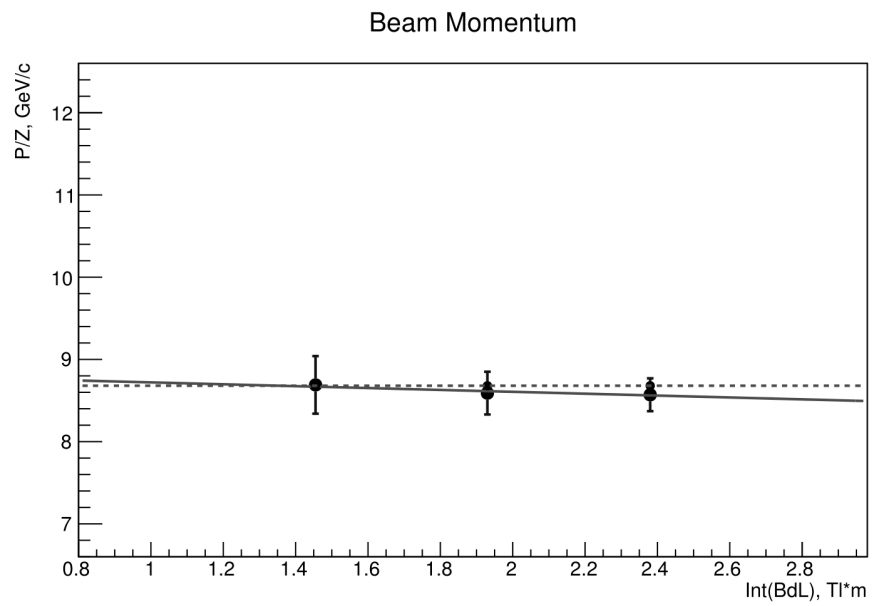

Figure 2. $\mathrm{C}$ beam energy $3.5 \mathrm{GeV} /$ nucleon momentum estimation. The dashed line is the nominal beam momentum value. The points are the beam momentum values estimated from experimental data.

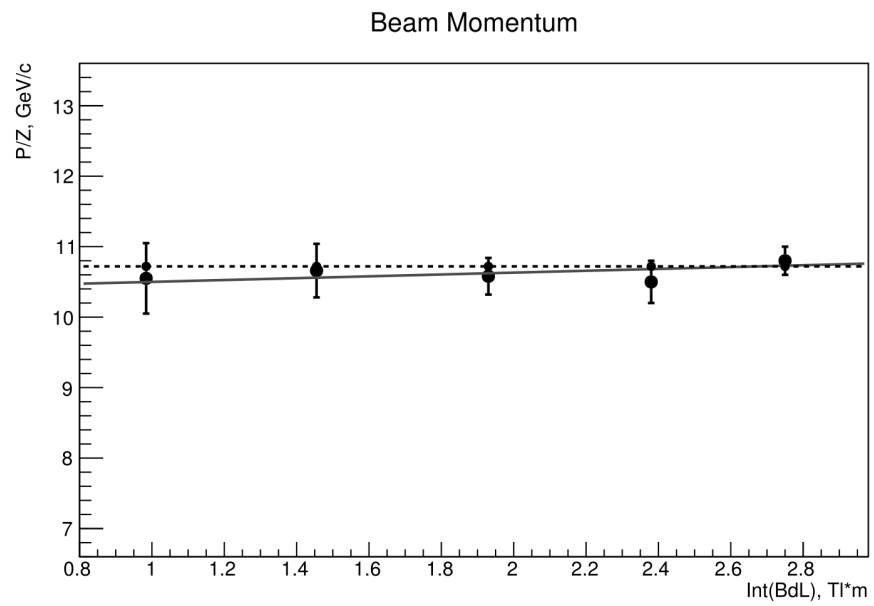

Figure 3. $\mathrm{C}$ beam energy $4.5 \mathrm{GeV} /$ nucleon momentum estimation. The dashed line is the nominal beam momentum value. The points are the beam momentum values estimated from experimental data. 
The dashed line represents the nominal beam momentum value calculated by the formula 2 and it is at $8.7 \mathrm{GeV} / \mathrm{c}$. The three points fitted with a straight solid line are the values estimated from the experimental data. They satisfy the nominal values within errors. The resolution gets improved with the increase of the magnetic field integrals values from $4 \%$ to $2.2 \%$. The obtained errors have contributions from different factors: the multiple scattering in the detectors' material and the precision with which the magnetic field, the incoming and the outcoming angles were measured. Minor influence has the multiple scattering in the material of the three tracking systems: MWPC, GEM and DCH. The magnetic field was measured by the accelerator team, but we do not have any information on its precision. The beam trajectory was positioned by the MWPC system at $0^{\circ}$ with an error less than $1 \%$. The outcoming angle values given by the DCH system increase with the increase of the magnetic field integral while their errors decrease from $4 \%$ for the lowest value of the integral down to $2 \%$ for the highest values of the field integral. The same procedure was performed for carbon beam with the energy of $4.5 \mathrm{GeV} /$ nucleon and the results are shown in figure 3. All the estimated values and their errors satisfy the expected results and the desired resolution of $2 \%$ was achieved for the working values of the magnetic field integral.

\section{Conclusion}

The BM@N experiment is operating and gathering experimental data as the first step of NICA project. All of its detector systems were commissioned and showed very good performance through last physical runs, in particular, the two detector systems described in detail in this paper demonstrated high efficiency and spatial resolution in track reconstruction. All these along with the global alignment procedure performed give us the possibility to estimate the beam momentum value with a precision of $2 \%$ for the working values of the magnetic field integral. The high precision and accuracy of the momentum estimation certifies that this system of detectors is advantageous for the track reconstruction in the sophisticated runs that involve targets and multiple scattering which are of the highest interest for the BM@N experiment.

\section{References}

[1] NICA Collaboration (Sissakian, A.N. et al.), J. Phys. G36 064069 (2009)

[2] M. Kapishin, Eur. Phys. J. A 52, 213-219 (2016)

[3] F. Sauli, Nucl. Instrum. Meth. A 805, 2-24 (2016)

[4] E. Lenivenko and V. Palichik, JINR preprint P1-2017-26 [in Rus.], 3-7 (2017)

[5] G.D. Barr et all, CERN/SPSC/90-22/P253

[6] R. Frühwirth, M. Regler, K. Bock, H. Grote, and D. Notz, Data Analysis Techniques for HighEnergy Physics (Cambridge Monographs on Particle Physics, Nuclear Physics and Cosmology, No. 11, 2nd ed., 2000) 